\title{
Impact of educational intervention on cervical cancer screening uptake among reproductive age women
}

\author{
Ajoke Akinola, Menira S. Constance*
}

Department of Public Health, Noida International University, Uttar Pradesh, India

Received: 12 January 2021

Revised: 11 February 2021

Accepted: 12 February 2021

\author{
*Correspondence: \\ Menira S. Constance, \\ E-mail: meniraconnie@gmail.com
}

Copyright: ( ) the author(s), publisher and licensee Medip Academy. This is an open-access article distributed under the terms of the Creative Commons Attribution Non-Commercial License, which permits unrestricted non-commercial use, distribution, and reproduction in any medium, provided the original work is properly cited.

\begin{abstract}
The objective of this review is to examine the efficacy of educational approaches in increasing women's adoption of cervical screening. A search was conducted through PubMed, Medline and Google Scholar. Articles that met the eligibility criteria were reviewed. The preferred reporting items for systematic reviews and meta-analysis (PRISMA) guided the data synthesis and reporting. We conducted a qualitative synthesis of evidence and pooled individual research results in meta-analyses using RevMan 5.3 review manager, where applicable. Heterogeneity has been estimated for total summary results of studies pooled together. Quality analysis of the papers was done using JADAD scale. This meta-analysis included a total of nine articles $(n=2316)$. Statistical research findings indicate that the use of cervical cancer educational approaches substantially improved cervical cancer screening rates and screening acceptability by double folds 2.88 . The results of this study support the use of educational intervention for cervical cancer to increase the acceptance of cervical cancer screening by women. We also recommend more research to assess the efficacy of various teaching approaches and the effect of intervention length on the rise in women's cervical cancer screening services utilization.
\end{abstract}

Keywords: Educational intervention, Cervical cancer, Screening, Reproductive age women

\section{INTRODUCTION}

\section{Global burden of cervical cancer}

Cervical cancer is the fourth most prevalent cancer in women worldwide, with an estimated 570,000 new cases in 2018, accounting for 7.5 percent of all deaths from female cancer. Of the estimated annual deaths of more than 311,000 from cervical cancer, more than $85 \%$ of these occur in low- and middle-income countries. ${ }^{1}$ Compared to women without human-immunodeficiency virus (HIV), women living with HIV are six times more likely to get cervical cancer, and an estimated 5 percent of all cases of cervical cancer are due to HIV. ${ }^{2}$ After breast cancer (2.1 million cases), colorectal cancer ( 0.8 million) and lung cancer (0.7 million), cervical cancer is listed. The worldwide median age-standard incidence of cervical cancer was 13.1 per 100,000 women, and this ranged widely across countries, with rates varying from less than 2 to 75 per 100,000 women. ${ }^{3}$

The leading cause of cancer-related death among women in eastern, western, central, and southern Africa is cervical cancer. The highest incidence was estimated in Eswatini, which had cervical cancer in around 6.5 percent of women before the age of 75 years. More than $1 / 3$ rd of the global cervical burden was contributed by both China and India, with 106000 and 97000 cases in China and India respectively, and 48000 deaths in China and 60000 deaths in India. The mean age for diagnosis of cervical cancer worldwide was 53 years, ranging from 44 years (Vanuatu) to 68 years (Vanuatu) (Singapore). The global mean age for cervical cancer deaths was 59 years, ranging from 45 years (Vanuatu) to 76 years (Vanuatu) (Martinique). In 146 
(79 percent) of the 185 countries analysed, cervical cancer ranked among the top three cancers affecting women younger than 45 years of age. ${ }^{4}$

70 percent of cervical cancers and pre-cancerous cervical lesions are caused by two human papillomavirus (HPV) types (16 and 18). If diagnosed at an early stage and rapidly treated, cervical cancer can be cured. ${ }^{5}$ The most common HPV-related illness is known to be cervical cancer. Nearly all cervical cancer cases may be related to HPV infection. ${ }^{6}$ Testing for high-risk HPV screening is recommended by the World Health Organization (WHO) and the European guidelines for quality assurance for cervical cancer screening. ${ }^{7,8}$ HPV testing has been shown to be successful in detecting precancerous cervical lesions, particularly in population-based cervical screening programs. ${ }^{9-12}$

A systematic study of cervical and breast cancer in 187 countries between 1980 and 2010 by Foremen et al found that developed countries with robust cancer screening programs have recorded steady decreases in cervical cancer incidence and mortality, whereas many developing countries have experienced increases in cervical cancer incidence in sub-Saharan Africa. ${ }^{13}$ Timely identification of precancerous cervical lesions by screening remains a crucial health care intervention for decreasing cervical cancer incidence and mortality, especially in low-resource settings where HPV vaccination coverage is limited, given that there are comprehensive campaigns to increase HPV vaccination uptake (Campos et al). ${ }^{9}$

WHO statistics and other studies show developed countries with well-regulated cervical cancer screening programs have achieved a substantial decline in cervical cancer incidence and mortality in relation to developing countries with low vaccine coverage and lack of organized cervical cancer screening programs. ${ }^{14-16}$ Indeed, evidence from Viriginia et al and CDC indicates that since Pap smear test originated, the incidence and mortality of cervical cancer in the USA has decreased with coordinated cervical cancer screening services and screening rates of $83 \%$ in the USA. ${ }^{17,18}$ However, in developed countries, where cervical cancer screening rates are currently low, ranging from 6-8 percent, cervical cancer remains a huge burden. ${ }^{19,20}$ These variations in screening rates and coverage for HPV vaccination could explain the differences in cervical cancer-related incidence and mortality in different regions around the world.

Evidence of the efficacy of cervical screening procedures has been reported in previous studies. ${ }^{21-23}$ One of the reviews centred on recognizing the effects of education on cervical cancer compared with control factors on screening rates for cervical cancer in qualifying women at risk of cervical cancer. It found that the use of theoretical educational approaches substantially increased CCS rates by more than twice as much as $(\mathrm{OR}=2.46,95$ percent $\mathrm{CI}$ : $1.88,3.21)$. In addition, giving women the option of selfsampling for testing for HPV increased CCS rates by almost 2 times $(\mathrm{OR}=1.71,95$ percent $\mathrm{CI}$ : $1.32,2.22)$. The research also found that invitation letters alone (or with a telephone follow-up contact), making an appointment, and sending reminders due or overdue for screening to patients had a significant impact on improving particle enhancement. Improvement of participation and CCS concentrations in at risk populations. ${ }^{24}$ The aim of this systematic review was therefore to better understand the latest evidence on the impact of education on cervical cancer as an intervention to increase screening rates of cervical cancer in women eligible for cervical cancer screening.

\section{Search strategy and eligibility criteria}

MEDLINE, PubMed and Google Scholar were searched for studies published in any country from 2005 to 2020 and published in English to answer the research question of this paper. The findings were limited to RCTs and quasiexperimental comparative community research involving women aged 15 years and older.

\section{Primary outcome}

At the end of the interventions, the findings of concern were cervical cancer screening uptake and willingness to take cervical cancer screening. In order to include all primary studies meeting the inclusion criteria, reference lists of on-topic systematic reviews were also searched.

\section{Study selection and data extraction}

All citations detected were screened for full text analysis, citations considered appropriate were retrieved. Full text papers were independently evaluated for eligibility, relevant outcome data, research information were extracted and entered under the headings Authors, year of publication and country, study design, sampling method, sample size, action, outcome and country in descriptive Table 1.

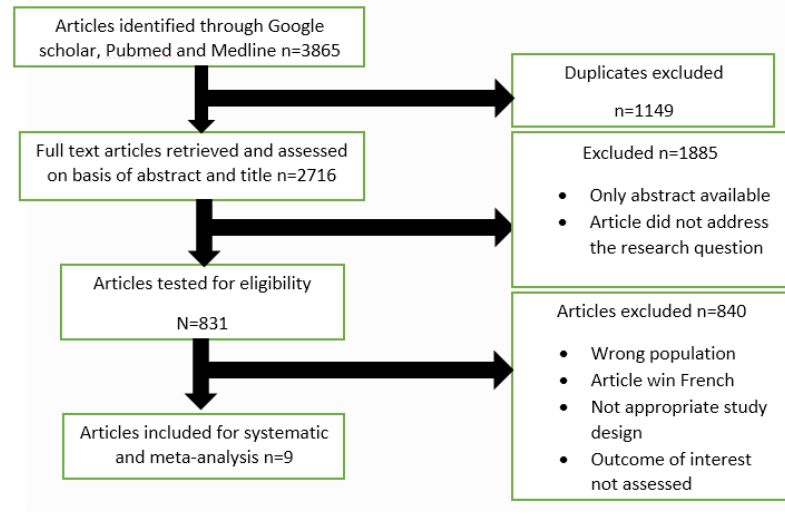

Figure 1: PRISMA diagram.

\section{Data synthesis}

The PRISMA statement guides the synthesis and reporting of the results. ${ }^{25} \mathrm{~A}$ brief narrative of the authors, years of 
publication, styles of intervention, environment, region, research population, key findings, and a description of the intervention effects and $p$ values for each study report were included in the qualitative synthesis. Using the RevMan 5.3 examination manager program, statistical pooling of the results of individual studies was achieved with metaanalysis. Specific forest plots for graphic display of the individual study effects and the overall summary effect of the interventions on cervical cancer screening rates were created by RevMan 5.3. Both statistical estimates of the individual and combined study effects of interventions in meta-analyses were developed using the odds ratio and random effects models.
This systematic and meta-analysis contains a total of 9 publications. Three studies by Tomas et al, Jenifer et al conducted in the USA and Joelle et al conducted in Kenya were RCT studies and the remaining six studies by Chizoma et al, Fang et al, Olumide et al, Parisa et al, Shabnan et al and Nancy et al used quasi experimental study design. ${ }^{26-34}$

The details of authors, study population, country, study design and sampling techniques used and outcome measures of intervention group and control group as well as the $\mathrm{p}$ values are entered in Table 2 .

Table 1: Descriptive table of studies selected.

\begin{tabular}{|c|c|c|c|c|c|c|}
\hline $\begin{array}{l}\text { Authors, } \\
\text { year of } \\
\text { publicat- } \\
\text { ion and } \\
\text { country }\end{array}$ & $\begin{array}{l}\text { Study } \\
\text { design }\end{array}$ & $\begin{array}{l}\text { Sampling } \\
\text { technique }\end{array}$ & $\begin{array}{l}\text { Samp } \\
\text {-le } \\
\text { size }\end{array}$ & Intervention & Result & $\begin{array}{l}\text { Conclusi- } \\
\text { on }\end{array}$ \\
\hline $\begin{array}{l}\text { Tomas et } \\
\text { al, 2010, } \\
\text { USA }^{25}\end{array}$ & $\mathrm{RCT}$ & $\begin{array}{l}\text { Random } \\
\text { sampling } \\
\text { method }\end{array}$ & 381 & $\begin{array}{l}\text { Social cognitive theory (SCT) was } \\
\text { used as a guiding principle for the } \\
\text { intervention design. All women } \\
\text { randomized to the intervention } \\
\text { were asked to attend at least one } \\
\text { teaching class and } 1 \text { year later were } \\
\text { given a refresher class. Women } \\
\text { received no educational } \\
\text { intervention in the normal care } \\
\text { group. All were sent a reminder by } \\
\text { mail and got a phone call reminder } \\
\text { about scheduling a visit for breast } \\
\text { and cervical screening }\end{array}$ & $\begin{array}{l}\text { Women in the } \\
\text { intervention group } \\
\text { were } 1.5 \text { times } \\
\text { more likely to } \\
\text { report getting a Pap } \\
\text { smear in the last } \\
\text { year, although this } \\
\text { was not statistically } \\
\text { significant ( } 95 \% \\
\text { CI=0.9-2.6) }\end{array}$ & $\begin{array}{l}\text { Educatio- } \\
\text { nal } \\
\text { interventio } \\
\mathrm{n} \text { based of } \\
\text { SCT model } \\
\text { was } \\
\text { effective }\end{array}$ \\
\hline $\begin{array}{l}\text { Joelle et } \\
\text { al, 2015, } \\
\text { Kenya }^{27}\end{array}$ & $\mathrm{RCT}$ & $\begin{array}{l}\text { Random } \\
\text { sampling }\end{array}$ & 467 & $\begin{array}{l}\text { An informative } 30 \text {-minute talk on } \\
\text { cervical cancer reviews basic health } \\
\text { information about cervical cancer, } \\
\text { risk factors, how screening is done, } \\
\text { what screening outcomes mean, } \\
\text { and options for treatment. A guided } \\
\text { discussion on screening barriers } \\
\text { and issues or stigma associated } \\
\text { with screening was included }\end{array}$ & $\begin{array}{l}\text { In the intervention } \\
\text { group, the } \\
\text { screening } \\
\text { acceptability of } \\
\text { women increased } \\
\text { but was not } \\
\text { substantially } \\
\text { different from the } \\
\text { initial sample } \\
(\mathrm{p}=0.26)\end{array}$ & $\begin{array}{l}\text { The } \\
\text { educatio- } \\
\text { nal } \\
\text { interventi- } \\
\text { on did not } \\
\text { lead to } \\
\text { higher } \\
\text { rates of } \\
\text { screening }\end{array}$ \\
\hline $\begin{array}{l}\text { Chizoma } \\
\text { et al, 2017, } \\
\text { Kenya }^{29}\end{array}$ & $\begin{array}{l}\text { Quasi } \\
\text { experi } \\
\text { mental }\end{array}$ & $\begin{array}{l}\text { Systematic } \\
\text { random } \\
\text { sampling }\end{array}$ & 904 & $\begin{array}{l}\text { Data on cervical cancer and } \\
\text { screening presented by the nurses } \\
\text { to women attending antenatal } \\
\text { clinics. On the clinical days in the } \\
\text { intervention group, health lessons } \\
\text { were given to the women's cluster } \\
\text { and they collected the knowledge in } \\
\text { groups. In supplying information } \\
\text { on cervical cancer in hospitals, } \\
\text { nurses used flex charts with } \\
\text { detailed information on cervical } \\
\text { cancer, women in the control group } \\
\text { were not exposed to such } \\
\text { information }\end{array}$ & $\begin{array}{l}\text { CCS uptake } \\
\text { increased } \\
\text { marginally from } \\
1.4 \% \text { at baseline to } \\
3.6 \% \text { at IG and } \\
2.1 \% \text { to } 2.3 \% \text { at } \\
\text { post intervention } \\
\text { CG }\end{array}$ & $\begin{array}{l}\text { Despite the } \\
\text { nurse-led } \\
\text { educatio- } \\
\text { nal } \\
\text { initiative, } \\
\text { cervical } \\
\text { cancer } \\
\text { screening } \\
\text { uptake } \\
\text { remained } \\
\text { low }\end{array}$ \\
\hline
\end{tabular}




\begin{tabular}{|c|c|c|c|c|c|c|}
\hline $\begin{array}{l}\text { Authors, } \\
\text { year of } \\
\text { publicat- } \\
\text { ion and } \\
\text { country }\end{array}$ & $\begin{array}{l}\text { Study } \\
\text { design }\end{array}$ & $\begin{array}{l}\text { Sampling } \\
\text { technique }\end{array}$ & $\begin{array}{l}\text { Samp } \\
\text {-le } \\
\text { size }\end{array}$ & Intervention & Result & $\begin{array}{l}\text { Conclusi- } \\
\text { on }\end{array}$ \\
\hline $\begin{array}{l}\text { Fang et al, } \\
2007 \text {, } \\
\text { USA }^{29}\end{array}$ & $\begin{array}{l}\text { Quasi } \\
\text { experi } \\
\text { mental }\end{array}$ & $\begin{array}{l}\text { Systematic } \\
\text { random } \\
\text { sampling }\end{array}$ & 102 & $\begin{array}{l}\text { Cervical cancer education and } \\
\text { patient navigation supported by } \\
\text { bilingual Korean health educators } \\
\text { were obtained by the intervention } \\
\text { group. The control group obtained } \\
\text { general health education, including } \\
\text { cervical cancer details and } \\
\text { screening }\end{array}$ & $\begin{array}{l}83 \% \text { of women in } \\
\text { the intervention } \\
\text { group had } \\
\text { undergone } \\
\text { screening at } 6 \\
\text { months post } \\
\text { intervention, } \\
\text { compared with } 22 \% \\
\text { in the control group }\end{array}$ & $\begin{array}{l}\text { Increased } \\
\text { screening } \\
\text { rates } \\
\text { observed }\end{array}$ \\
\hline $\begin{array}{l}\text { Olumide } \\
\text { et al, 2014, } \\
\text { Nigeria }^{30}\end{array}$ & $\begin{array}{l}\text { Quasi } \\
\text { experi } \\
\text { mental }\end{array}$ & $\begin{array}{l}\text { Multistage } \\
\text { sampling } \\
\text { technique }\end{array}$ & 700 & $\begin{array}{l}\text { A film on cervical cancer and } \\
\text { screening was used by the health } \\
\text { education intervention to stimulate } \\
\text { participatory health education. } \\
\text { Hand bills created in both Yoruba } \\
\text { (the local language) and English } \\
\text { were given to reinforce what had } \\
\text { been taught. Period of the 7-day } \\
\text { intervention }\end{array}$ & $\begin{array}{l}\text { A statistically } \\
\text { significant } \\
\text { difference between } \\
\text { the intervention and } \\
\text { control groups in } \\
\text { their attitude toward } \\
\text { cervical and } \\
\text { screening } \\
\text { awareness and } \\
\text { practice ( } \mathrm{p}<0.05) \\
\text { after the } \\
\text { intervention was } \\
\text { recorded }\end{array}$ & $\begin{array}{l}\text { Health } \\
\text { education } \\
\text { interventio } \\
n \text { was } \\
\text { effective }\end{array}$ \\
\hline $\begin{array}{l}\text { Parisa et } \\
\text { al, 2017, } \\
\text { Iran }^{31}\end{array}$ & $\begin{array}{l}\text { Quasi } \\
\text { experi } \\
\text { mental }\end{array}$ & $\begin{array}{l}\text { Multistage } \\
\text { sampling } \\
\text { technique }\end{array}$ & 80 & $\begin{array}{l}\text { The intervention was carried out as } \\
\text { community counselling based on } \\
\text { the Health Belief Model and } \\
\text { Collect counselling measures in the } \\
\text { intervention group. Three } 45-60 \\
\text { min sessions with a one-week } \\
\text { interval and a capacity of } 10 \text { people } \\
\text { per session were conducted during } \\
\text { the counselling meeting, using the } \\
\text { community counselling approach } \\
\text { with posters and pamphlets in } \\
\text { village health houses in the } \\
\text { intervention group }\end{array}$ & $\begin{array}{l}\text { The Pap smear } \\
\text { examination was } \\
\text { conducted by } 17 \\
\text { patients from the } \\
\text { intervention group } \\
\text { and } 4 \text { patients from } \\
\text { the control group } \\
\text { after the } \\
\text { intervention } \\
(\mathrm{p}<0.055)\end{array}$ & $\begin{array}{l}\text { The group } \\
\text { counselling } \\
\text { based on } \\
\text { the health } \\
\text { belief } \\
\text { mode was } \\
\text { effective }\end{array}$ \\
\hline $\begin{array}{l}\text { Nancy et } \\
\text { al, 2019, } \\
\text { Ghana }^{34}\end{array}$ & $\begin{array}{l}\text { Non- } \\
\text { equival } \\
\text {-ent } \\
\text { control- } \\
\text { group } \\
\text { design }\end{array}$ & $\begin{array}{l}\text { Convenien } \\
\text {-ce } \\
\text { sampling } \\
\text { technique }\end{array}$ & 782 & $\begin{array}{l}\text { Health education provided cervical } \\
\text { cancer information and screening to } \\
\text { boost the level of awareness of the } \\
\text { disease. Focus of cervical cancer } \\
\text { education was on the cause, } \\
\text { predisposing factors, } \\
\text { signs/symptoms, complications and } \\
\text { prevention methods. With regard to } \\
\text { cervical cancer screening, } \\
\text { individuals were introduced to } \\
\text { where they could go for testing }\end{array}$ & $\begin{array}{l}\text { Despite the } \\
\text { obstacles to cervical } \\
\text { cancer screening, } \\
\text { the intervention } \\
\text { community showed } \\
\text { high awareness and } \\
\text { optimistic beliefs } \\
\text { about cervical } \\
\text { cancer screening }\end{array}$ & $\begin{array}{l}\text { Health } \\
\text { education- } \\
\mathrm{n} \text { is } \\
\text { effective }\end{array}$ \\
\hline $\begin{array}{l}\text { Shabnam } \\
\text { et al, 2017, } \\
\text { Iran }^{32}\end{array}$ & $\begin{array}{l}\text { Quasi- } \\
\text { experi } \\
\text { mental } \\
\text { study } \\
\text { design }\end{array}$ & $\begin{array}{l}\text { Cluster and } \\
\text { simple } \\
\text { random } \\
\text { sampling } \\
\text { techniques } \\
\text { were used }\end{array}$ & 143 & $\begin{array}{l}\text { The experimental group received an } \\
\text { educational program on cervical } \\
\text { cancer focused on the PMT model. } \\
\text { Every } 45 \text {-minute session was } \\
\text { planned for small groups of } 10 \text { to } \\
15 \text { women. During the sessions, } \\
\text { after each educational session, the } \\
\text { participants were educated on the } \\
\text { basis of active learning methods, }\end{array}$ & $\begin{array}{l}\text { The incidence of } \\
\text { routine Pap smear } \\
\text { examination and } \\
\text { referral to health } \\
\text { centres was } \\
\text { significantly } \\
\text { increased } \\
(\mathrm{p}=0.048) \text {, but not }\end{array}$ & $\begin{array}{l}\text { PMT- } \\
\text { based } \\
\text { educational } \\
\text { interventio } \\
\text {-n was } \\
\text { successful } \\
\text { in } \\
\text { enhancing } \\
\text { periodic }\end{array}$ \\
\hline
\end{tabular}




\begin{tabular}{|c|c|c|c|c|c|c|}
\hline $\begin{array}{l}\text { Authors, } \\
\text { year of } \\
\text { publicat- } \\
\text { ion and } \\
\text { country }\end{array}$ & $\begin{array}{l}\text { Study } \\
\text { design }\end{array}$ & $\begin{array}{l}\text { Sampling } \\
\text { technique }\end{array}$ & $\begin{array}{l}\text { Samp } \\
\text {-le } \\
\text { size }\end{array}$ & Intervention & Result & $\begin{array}{l}\text { Conclusi- } \\
\text { on }\end{array}$ \\
\hline & & & & $\begin{array}{l}\text { including lectures, group } \\
\text { discussions and questions-answers } \\
\text { and pamphlets and a booklet were } \\
\text { given to women }\end{array}$ & $\begin{array}{l}\text { in the control group } \\
(\mathrm{p}>0.05)\end{array}$ & $\begin{array}{l}\text { Pap smear } \\
\text { testing }\end{array}$ \\
\hline $\begin{array}{l}\text { Molokwu } \\
\text { et al, 2018, } \\
\text { USA }^{26}\end{array}$ & $\begin{array}{l}\text { Rando- } \\
\text { mized } \\
\text { control- } \\
\text { ed trial }\end{array}$ & $\begin{array}{l}\text { Random } \\
\text { sampling } \\
\text { technique }\end{array}$ & 201 & $\begin{array}{l}\text { The intervention group receives } \\
\text { culturally tailored education from } \\
\text { the promoter while the control } \\
\text { group receives a written training } \\
\text { pamphlet }\end{array}$ & $\begin{array}{l}\text { The study found a } \\
\text { high degree of self- } \\
\text { sampling } \\
\text { acceptability } \\
\text { independent of } \\
\text { educational severity } \\
\text { and a trend towards } \\
\text { increased follow-up } \\
\text { of Pap smear in } \\
\text { participants who } \\
\text { tested positive }\end{array}$ & $\begin{array}{l}\text { No } \\
\text { significant } \\
\text { difference }\end{array}$ \\
\hline
\end{tabular}

Table 2: Descriptive table of studies selected.

\begin{tabular}{|c|c|c|c|c|c|c|c|c|c|}
\hline Author & Population & Country & Year & $\begin{array}{l}\text { Study } \\
\text { design }\end{array}$ & $\begin{array}{l}\text { Sampling } \\
\text { method }\end{array}$ & $\begin{array}{l}\text { Intervention } \\
\text { group } \\
\text { (screened) }\end{array}$ & $\begin{array}{l}\text { Control } \\
\text { group } \\
\text { (screened) }\end{array}$ & $\begin{array}{l}\mathbf{P} \\
\text { value }\end{array}$ & $\begin{array}{l}\text { Study } \\
\text { quality }\end{array}$ \\
\hline $\begin{array}{l}\text { Tomas et } \\
\text { al }^{25}\end{array}$ & $\begin{array}{l}\text { Women } \\
\geq 50 \text { years }\end{array}$ & USA & 2010 & $\mathrm{RCT}$ & $\begin{array}{l}\text { Random } \\
\text { sampling }\end{array}$ & $93 / 104$ & $87 / 116$ & 0.05 & High \\
\hline $\begin{array}{l}\text { Joelle et } \\
\mathrm{al}^{27}\end{array}$ & Women & Kenya & 2015 & $\mathrm{RCT}$ & $\begin{array}{l}\text { Random } \\
\text { sampling }\end{array}$ & $145 / 161$ & $155 / 167$ & 0.37 & High \\
\hline $\begin{array}{l}\text { Chizoma } \\
\text { et } \mathbf{a l}^{28}\end{array}$ & Women & Nigeria & 2017 & $\begin{array}{l}\text { Quasi } \\
\text { experim- } \\
\text { mental }\end{array}$ & $\begin{array}{l}\text { Systematic } \\
\text { random } \\
\text { sampling }\end{array}$ & $15 / 417$ & $10 / 429$ & 0.27 & High \\
\hline $\begin{array}{l}\text { Fang et } \\
\text { al }^{29}\end{array}$ & Women & USA & 2007 & $\begin{array}{l}\text { Quasi } \\
\text { experim- } \\
\text { mental }\end{array}$ & $\begin{array}{l}\text { Random } \\
\text { sampling }\end{array}$ & $39 / 46$ & $5 / 39$ & $<0.001$ & High \\
\hline $\begin{array}{l}\text { Olumide } \\
\text { et } \mathbf{a l}^{30}\end{array}$ & $\begin{array}{l}\text { Women } 25- \\
64\end{array}$ & Nigeria & 2014 & $\begin{array}{l}\text { Quasi } \\
\text { experim- } \\
\text { mental }\end{array}$ & $\begin{array}{l}\text { Multistage } \\
\text { sampling }\end{array}$ & $300 / 325$ & $270 / 289$ & 0.038 & High \\
\hline $\begin{array}{l}\text { Parisa et } \\
\mathrm{al}^{31}\end{array}$ & Women & Iran & 2017 & $\begin{array}{l}\text { Quasi } \\
\text { experim- } \\
\text { mental }\end{array}$ & $\begin{array}{l}\text { Multistage } \\
\text { sampling }\end{array}$ & $37 / 40$ & $16 / 40$ & $<0.001$ & High \\
\hline $\begin{array}{l}\text { Shabnam } \\
\text { et } \mathbf{a l}^{32}\end{array}$ & Women & Iran & 2017 & $\begin{array}{l}\text { Quasi } \\
\text { experim- } \\
\text { mental }\end{array}$ & $\begin{array}{l}\text { Cluster } \\
\text { and simple } \\
\text { random } \\
\text { sampling }\end{array}$ & $35 / 72$ & $23 / 71$ & 0.048 & High \\
\hline
\end{tabular}

\section{Quality assessment}

The JADAD quality assessment scale was used. It has three measures which is randomization, to which 1 score is given if the study described as randomized and an additional point if the method for generating the sequence of randomization was described and it was appropriate and 1 point is deducted if the method for generating the sequence of randomization was described and it was inappropriate. 1 point if the study described as double blind and extra 1 point if the method of double-blinding was described and it was appropriate and 1 point deducted if the method of double-blinding was described and it was inappropriate. The third item on the scale assesses if the studies have a description of withdrawals and dropouts (30). *Yes $=1$, for a total of 5 possible points; $\geq 3$ points indicate a superior quality trial. The 6 studies which meet the eligibility have a score of $\geq 3$ and are considered as high quality. Details of studies with score of greater or equal to three are included in Table 3.

A search through Google scholar, Pubmed and Medline yielded 3865 articles, after duplicates were removed, a total of 2716 full text articles were retrieved and assessed 
on basis of abstract and title. Of the 831 articles assessed for eligibility, only 9 articles were included in qualitative synthesis. To respond to the research question, "What is the effect of educational intervention of cervical cancer screening rates and acceptability of cervical cancer screening?" seven studies were put together. Three random controlled studies (RCT) and four quasi experimental studies. Three (3) of the studies were conducted from USA and two from Nigeria, two from Iran and one from Kenya.

Table 3: Details of studies with score of greater or equal to three.

\begin{tabular}{|lllll|}
\hline Study & $\begin{array}{l}\text { Random- } \\
\text { ization }\end{array}$ & $\begin{array}{l}\text { Blind } \\
\text {-ing }\end{array}$ & $\begin{array}{l}\text { Description } \\
\text { of } \\
\text { withdrawals }\end{array}$ & $\begin{array}{l}\% \\
\text { max } \\
\text { score }\end{array}$ \\
\hline $\mathbf{2 6}$ & 2 & 1 & 1 & 4 \\
\hline $\mathbf{2 8}$ & 2 & 1 & 1 & 4 \\
\hline $\mathbf{2 9}$ & 2 & 1 & 0 & 3 \\
\hline $\mathbf{3 0}$ & 2 & 1 & 1 & 4 \\
\hline $\mathbf{3 1}$ & 2 & 1 & 0 & 3 \\
\hline $\mathbf{3 2}$ & 2 & 1 & 2 & 5 \\
\hline $\mathbf{3 3}$ & 2 & 1 & 1 & 4 \\
\hline
\end{tabular}

\begin{tabular}{|c|c|c|c|c|c|c|c|c|c|}
\hline \multirow[b]{2}{*}{ Study or Subgroup } & \multicolumn{2}{|c|}{ Experimental } & \multirow[t]{2}{*}{ Control } & \multirow{2}{*}{\multicolumn{3}{|c|}{$\begin{array}{c}\text { Odds Ratio } \\
\text { Weight } \mathrm{M} \cdot \mathrm{H} \text {, Random, } 95 \% \mathrm{Cl}\end{array}$}} & \multirow{2}{*}{\multicolumn{3}{|c|}{$\begin{array}{c}\text { Odds Ratio } \\
\text { M.H, Random, } 95 \% \mathrm{Cl}\end{array}$}} \\
\hline & Events & Total & & & & & & & \\
\hline Carolyn 2007 & 39 & 46 & $\begin{array}{ll}5 & 39\end{array}$ & $12.6 \%$ & $37.89[11.00,130.44]$ & & & & $\vec{t}$ \\
\hline Chizoma 2017 & 15 & 417 & $10 \quad 429$ & $14.7 \%$ & $1.56[0.69,3.52]$ & & & & \\
\hline Joelle 2015 & 145 & 161 & $155 \quad 167$ & $14.8 \%$ & $0.70[0.32,1.53]$ & & & & \\
\hline Olumide 2014 & 300 & 325 & $270 \quad 289$ & $15.5 \%$ & $0.84[0.45,1.57]$ & & & & \\
\hline Parisa 2017 & 37 & 40 & $16 \quad 40$ & $12.1 \%$ & $18.50[4.86,70.36]$ & & & & \\
\hline Shabnum 2017 & 35 & 72 & $23 \quad 31$ & $15.3 \%$ & $1.97[1.00,3.89]$ & & & & \\
\hline Tomas 2010 & 93 & 104 & $87 \quad 116$ & $15.0 \%$ & $2.82[1.33,5.98]$ & & & & \\
\hline Total $(95 \% \mathrm{Cl})$ & & 1165 & 1151 & $100.0 \%$ & $2.88[1.19,6.98]$ & & & & \\
\hline Total events & 664 & & 566 & & & & & & \\
\hline $\begin{array}{l}\text { Heterogeneity. Tau" } \\
\text { Test for overall effec }\end{array}$ & $\begin{array}{l}1.21 ; \mathrm{Chi}^{2} \\
z=2.35(\mathrm{r}\end{array}$ & & $d f=6(P<$ & & & 0.0 & $\begin{array}{c}1 \\
0.1 \\
\text { Favours [control] }\end{array}$ & Favou & $\begin{array}{ll}10 & 100 \\
10 & \end{array}$ \\
\hline
\end{tabular}

Figure 2: Evidence of an improvement in screening rates for cervical cancer in women exposed to the intervention relative to the controls.

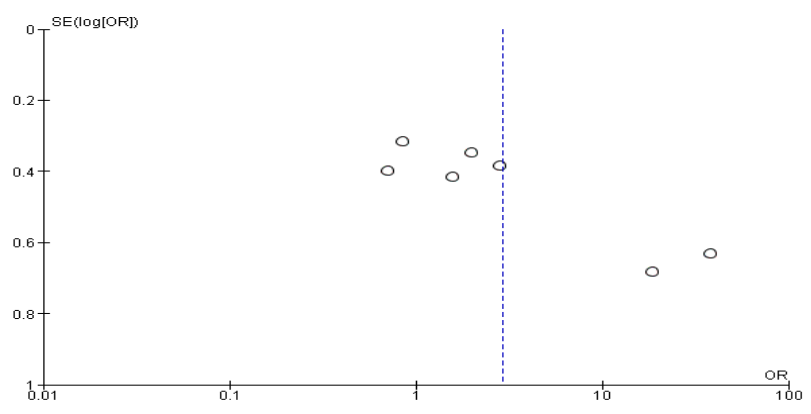

Figure 3: The Funnel plot.

In a meta-analysis of a total of 1165 women who were exposed to cervical cancer education and 1151 women in the reference community, seven studies were pooled together. The findings of our meta-analysis presented in Figure 2 showed evidence of an improvement in screening rates for cervical cancer in women exposed to the intervention relative to the controls. In the interventional group, the combined summary effect of the interventions included was two times greater than in the control group $2.88[1.19,6.98]$.

\section{DISCUSSION}

The key findings of this study are that educational approaches used in various communities to increase the acceptance of cervical cancer screening are successful. Cervical cancer and HPV procedures greatly increase cervical cancer screening rates. The forest plot above illustrates the cumulative results of seven studies on educational approaches for cervical cancer. This showed an average impact of 2.88. Women in the intervention group are twice likely to screen compared to the control group.

\section{Cervical cancer education}

Different methods were used to deliver cervical cancer education to study participants randomized into the interventional group. These interventions range from, the use social cognitive theory (SCT), interactive talk about cervical cancer, focused information on cervical cancer, use of movies on cervical cancer, one adopted the health belief model and GATHER counselling steps and other interventions delivered in bi-languages.

The social cognitive structure was used to direct one of the studies examined. This theory states that the perception of health risks and benefits is the precondition for improvement, and if people do not have knowledge of how their lifestyle behaviours relate to their health, they may have little reasons to support activities or attempts to change those unhealthy habits. ${ }^{26,35,36}$ In contrast with the normal treatment group, women in the intervention group were 1.5 times more likely to report getting a Pap smear during the last year. Community therapy focused on the wellbeing belief model and GATHER counselling measures was used in another report. Three 45-60 min sessions with a one-week interval and a capacity of 10 people per session were conducted during the counselling meeting, using the community counselling approach with posters and pamphlets in the village health houses of the intervention group. The health behaviour structure involves the combined impact of individual and health care system variables, environmental and personal barriers, a review by Musa et al. ${ }^{24,32}$ As was seen in the intervention groups of the studies included in their analysis, the use of theory-based educational approaches is very relevant for emerging communities with low levels of literacy, which is also similar to the results of this research. Our studies have shown a positive effect of educational involvement in increasing the acceptance of cervical cancer screening. Such initiatives not only increased women's cervical cancer screening rates, but also strengthened visibility, knowledge of cervical cancer, screening significance, and provided barrier advice and guidance on when and how to do CCS, thus improving the overall chances of qualified women turning up or scheduling cervical cancer screening. ${ }^{26,31,32}$ We have faith in the findings and 
encourage the use of educational approaches to improve women's participation in cervical screening programs, because only high-quality studies have been checked.

\section{Limitations}

This review only included studies retrieved from three electronic databases, results from studies published in other data bases could be different. More so, through the application of our inclusion criteria, articles excluded might give different results.

\section{CONCLUSION}

The findings of this review contribute to the growing literature supporting the implementation of educational interventions for cervical cancer to increase cancer screening rates among eligible women, particularly by targeting sexually active young people/students and illiterate women. Educational approaches such as social cognitive theory and the health belief system focused on theory-based driven cervical cancer are modelled to target frameworks such as avoiding screening for cervical cancer where no signs manifest, believing that it was easier to have no awareness of one's diagnosis of cervical cancer and believing that only women who participate in sexual risk-taking activities such as prostitution, drug abuse and polyandry need to receive pap smear and assist target groups to make informed health choices and facilitate the recognition and completion of cervical cancer screening activities. More research should, however, be carried out to assess the efficacy of provider recommendation initiatives, such as invitation letters with follow-up telephone call reminders, community advice and film use, suggestion and promotion of women's self-sampling methods to achieve a substantial improvement in screening rates. In order to provide more understanding and draw effective conclusions, the implications of the length of educational programs on cervical cancer screening rates should also be studied.

\section{Funding: No funding sources \\ Conflict of interest: None declared \\ Ethical approval: Not required}

\section{REFERENCES}

1. Latest global cancer data: Cancer burden rises to 18.1 million new cases and 9.6 million cancer deaths in 2018. Available at: https://www.who.int/cancer /PRGlobocanFinal.pdf. Accessed on 07 January 2021.

2. WHO releases new estimates of the global burden of cervical cancer associated with HIV. Available at: https://www.who.int/news/item/16-11-2020-whoreleases-new-estimates-of-the-global-burden-ofcervical-cancer-associated-with-hiv. Accessed on 07 January 2021.

3. Arbyn M, Weiderpass E, Bruni L, de Sanjosé S, Saraiya M, Ferlay J, Bray F. Estimates of incidence and mortality of cervical cancer in 2018: a worldwide analysis. Lancet Glob Health. 2020;8(2):191-203.

4. Lei J, Ploner A, Elfström KM, Wang J, Roth A, Fang $F$, et al. HPV Vaccination and the Risk of Invasive Cervical Cancer. N Engl J Med. 2020;383(14):13408.

5. Human papillomavirus (HPV) and cervical cancer. Available at: https://www.who.int/news-room/factsheets/detail/human-papillomavirus-(hpv)-andcervical-cancer. Accessed on 07 January 2021.

6. WHO guidelines WHO guidelines for screening and treatment of precancerous lesions for cervical cancer prevention. Available at: https://www.who.int/ reproductivehealth/publications/cancers/screening_a nd_treatment_of_precancerous_lesions/en/.

Accessed on 07 January 2021

7. Arbyn M, Anttila A, Jordan J, Ronco G, Schenck U, Segnan N, et al. European Guidelines for Quality Assurance in Cervical Cancer Screening. Second edition--summary document. Ann Oncol. 2010;21(3):448-58.

8. Campos NG, Tsu V, Jeronimo J, Mvundura M, Kim JJ. Evidence-based policy choices for efficient and equitable cervical cancer screening programs in lowresource settings. Cancer Med. 2017;6(8):2008-14.

9. Cremer ML, Conlisk E, Felix JC. HPV screening for cervical cancer in rural India. $\mathrm{N}$ Engl $\mathrm{J}$ Med. 2009;361(3):305

10. Ronco G, Dillner J, Elfström KM, Tunesi S, Snijders PJ, Arbyn M, et al. Efficacy of HPV-based screening for prevention of invasive cervical cancer: follow-up of four European randomised controlled trials. Lancet. 2014;383(9916):524-32.

11. Rijkaart DC, Berkhof J, Rozendaal L, van Kemenade FJ, Bulkmans NW, Heideman DA, et al. Human papillomavirus testing for the detection of high-grade cervical intraepithelial neoplasia and cancer: final results of the POBASCAM randomised controlled trial. Lancet Oncol. 2012;13(1):78-88.

12. Forouzanfar MH, Foreman KJ, Delossantos AM, Lozano R, Lopez AD, Murray CJ, Naghavi M. Breast and cervical cancer in 187 countries between 1980 and 2010: a systematic analysis. Lancet. 2011;378(9801):1461-84

13. Allemani C, Weir HK, Carreira H, Harewood R, Spika D, Wang XS, et al. Global surveillance of cancer survival 1995-2009: analysis of individual data for $25,676,887$ patients from 279 populationbased registries in 67 countries (CONCORD-2). Lancet. 2015;385(9972):977-1010.

14. Miller JW, Royalty J, Henley J, White A, Richardson LC. Breast and cervical cancers diagnosed and stage at diagnosis among women served through the National Breast and Cervical Cancer Early Detection Program. Cancer Causes Control. 2015;26(5):741-7.

15. White MC, Wong FL. Preventing premature deaths from breast and cervical cancer among underserved women in the United States: insights gained from a national cancer screening program. Cancer Causes Control. 2015;26(5):805-9. 
16. Smith RA, Cokkinides V, Brooks D, Saslow D, Shah M, Brawley OW. Cancer screening in the United States, 2011: A review of current American Cancer Society guidelines and issues in cancer screening. CA Cancer J Clin. 2011;61(1):8-30.

17. Moyer VA; U.S. Preventive Services Task Force. Screening for oral cancer: U.S. Preventive Services Task Force recommendation statement. Ann Intern Med. 2014;160(1):55-60.

18. Idowu A, Olowookere SA, Fagbemi AT, Ogunlaja OA. Determinants of Cervical Cancer Screening Uptake among Women in Ilorin, North Central Nigeria: A Community-Based Study. J Cancer Epidemiol. 2016;6469240.

19. Sudenga SL, Rositch AF, Otieno WA, Smith JS. Knowledge, attitudes, practices, and perceived risk of cervical cancer among Kenyan women: brief report. Int J Gynecol Cancer. 2013;23(5):895-9.

20. Everett T, Bryant A, Griffin MF, Martin-Hirsch PP, Forbes CA, Jepson RG. Interventions targeted at women to encourage the uptake of cervical screening. Cochrane Database Syst Rev. 2011;5:CD002834.

21. Baron RC, Melillo S, Rimer BK, Coates RJ, Kerner $\mathrm{J}$, Habarta $\mathrm{N}$, et al. Intervention to increase recommendation and delivery of screening for breast, cervical, and colorectal cancers by healthcare providers a systematic review of provider reminders. Am J Prev Med. 2010;38(1):110-7.

22. Everett T, Bryant A, Griffin MF, Martin-Hirsch PP, Forbes CA, Jepson RG. Interventions targeted at women to encourage the uptake of cervical screening. Cochrane Database Syst Rev. 2011;5:CD002834.

23. Musa J, Achenbach CJ, O'Dwyer LC, Evans CT, McHugh M, Hou L, et al. Effect of cervical cancer education and provider recommendation for screening on screening rates: A systematic review and meta-analysis. PLoS One. 2017;12(9):e0183924.

24. Liberati A, Altman DG, Tetzlaff J, Mulrow C, Gøtzsche PC, Ioannidis JPA, et al. The PRISMA statement for reporting systematic reviews and metaanalyses of studies that evaluate health care interventions: explanation and elaboration. J Clin Epidemiol. 2009;62(10):1-34.

25. Nuño T, Martinez ME, Harris R, García F. A Promotora-administered group education intervention to promote breast and cervical cancer screening in a rural community along the U.S.Mexico border: A randomized controlled trial. Cancer Causes Control. 2011;22(3):367-74.

26. Molokwu JC, Penaranda E, Dwivedi A, Mallawaarachchi I, Shokar N. Effect of educational intervention on self-sampling acceptability and follow-up paps in border dwelling hispanic females. J Low Genit Tract Dis. 2018;22(4):295-301.

27. Rosser JI, Njoroge B, Huchko MJ. Changing knowledge, attitudes, and behaviors regarding cervical cancer screening: The effects of an educational intervention in rural Kenya. Patient Educ Couns. 2015;98(7):884-9.

28. Ndikom CM, Ofi BA, Omokhodion FO, Adedokun BO. Effects of educational intervention on women's knowledge and uptake of cervical cancer screening in selected hospitals in Ibadan, Nigeria. Int $\mathbf{J}$ Health Promot Educ. 2017;55:5-6.

29. Fang CY, Ma GX, Tan Y, Chi N. A multifaceted intervention to increase cervical cancer screening among underserved Korean women. Cancer Epidemiol Biomarkers Prev. 2007;16(6):1298-302.

30. Abiodun OA, Olu-Abiodun OO, Sotunsa JO, Oluwole FA. Impact of health education intervention on knowledge and perception of cervical cancer and cervical screening uptake among adult women in rural communities in Nigeria. BMC Public Health. 2014;14(1):814.

31. Parsa P, Sharifi F, Shobeiri F, Karami M. Effects of group counseling based on health belief model on cervical cancer screening beliefs and performance of rural women in Kaboudrahang, Iran. Asian Pacific J Cancer Prev. 2017;18(6):1525-30.

32. Malmir S, Barati M, Jeihooni AK, Bashirian S, Hazavehei SMM. Effect of an educational intervention based on Protection Motivation Theory on preventing cervical cancer among marginalized women in west Iran. Asian Pacific J Cancer Prev. 2018;19(3):755-61.

33. Ebu NI, Amissah-Essel S, Asiedu C, Akaba S, Pereko KA. Impact of health education intervention on knowledge and perception of cervical cancer and screening for women in Ghana. BMC Public Health. 2019;19(1):1505.

34. Bandura A. Health promotion by social cognitive means. Health Educ Behav. 2004;31(2):143-64.

35. Social foundations of thought and action: A social cognitive theory. - PsycNET. Available at: https://psycnet.apa.org/record/1985-98423-000. Accessed on 07 January 2021.

Cite this article as: Constance MS, Akinola A. Impact of educational intervention on cervical cancer screening uptake among reproductive age women. Int J Community Med Public Health 2021;8:2053-60. 